\title{
Ten Open Challenges at the Boundaries of Software Engineering and Information Systems
}

\author{
Anthony Finkelstein \\ Department of Computer Science, University College London, UK
}

\begin{abstract}
In this talk, intended to provoke discussion, I will suggest ten important open challenges at boundaries where Software Engineering \& Information Systems meet. I will focus on challenges are both intellectually demanding and of industrial importance. I will suggest some approaches to meeting these challenges and will lay stress upon the interdisciplinary opportunities they give rise to.
\end{abstract}

